\title{
Inherited metabolic disorders - from genes to cell to patient to population to society and back
}

Annals of Translation Medicine (ATM) focused issue: inborn errors of metabolism (IEM) is a one stop resource for all levels of learners from undergraduates interested in science to multiple subspecialties in the field of medicine, genetics and public health. From the historical perspective of the term "Inborn Errors of Metabolism" coined by Sir Archibald Garrod connected biochemistry, clinical medicine and genetics more than 100 years ago. Subsequent historic IEM developments 50 years ago included Robert Guthrie's invention of phenylketonuria (PKU) test, Robert Macready's implementation of it as a public health program and the Kennedy family's political support. But, only current advances in molecular analysis and technology changed our understanding of previously considered "rare" IEM disorders with prevalence of less than 1 in 100,000 to 1 in 1,000 as a group.

This issue highlights the historical perspectives, biochemistry, genetics, clinical medicine, therapeutic advances. It furthermore connects the role of metabolic nutrition, public health and behavioral health to illustrate the translational concept-molecular biology to patient to population to behavior and its application back to population, patient and molecular levels. It attempts to show that the burden of such diseases not only affects the individual patient, but also impacts family, primary care providers, educational systems, public resources, public policies, research advances and ethics. With our focus towards a wide readership, the chapters are subdivided into commonly known IEM categories like 'intoxication', 'energy metabolism' and 'complex molecules' disorders. Furthermore, the twelve chapters highlight Newborn Screening, common diagnostic pearls for primary care providers, laboratory approaches to IEM apart from reviewing individual disorders within each subcategory such as amino acid disorder, organic acid disorders, fatty acid oxidation disorders, mitochondrial disorders, glycogen storage disorders, lysosomal storage disorders and congenital disorders of glycosylation. We have further elaborated on the importance of integrated behavioral health (IBH) model of care for optimal outcomes in patients, especially, since Newborn Screening is changing the natural history of previously known devastating conditions. Though each subcategory of disorders is approached from a general biochemistry and pathogenesis view point, we further detail each disorder specific - clinical characteristics, biochemical pathway defects, diagnostic metabolic findings, genetics and epidemiology, and available treatments. Authors have also provided their insight and thoughts on future directions in advancing the field. The use of supplemental figures and tables are quick helpful resources providing easy knowledge of various rare disorders and connections of various biochemical pathways.

While this issue is meant as a quick easy to read referral resource for all clinicians (primary care and subspecialists), medical students, public health personnel, researchers, basic science students and leaders, policy makers and ethicists to further their understanding on various IEMs, it also provides a glimpse on various aspects of this highly specialized medical field in the US. We strongly advocate that this issue be used in conjunction to discussions with local IEM clinicians for individual cases. IEM scientific organization members, though few, are wide spread all over the world in every continent, helping each other like a big family, ensuring IEM patients are never alone.

This issue is a tribute to (I) advocacy efforts of mothers guiding our initial diagnosis of PKU and the initial treatment strategy restricting the offending nutrient; (II) advocacy efforts of a sister, as Eunice Kennedy, in helping future IEM related public health and research endeavors; and (III) teachers like Professors Carol Greene and Georgianne Arnold nurturing future fellows and faculty in Biochemical Genetics.

I am privileged to get guidance of different IEM experts, participation and contribution of all of the authors helping bring this issue together.

\section{Acknowledgements}

My greatest thanks to my mother and Eloise grams for their encouragement to share my passion of IEMs to all learners around the world. 


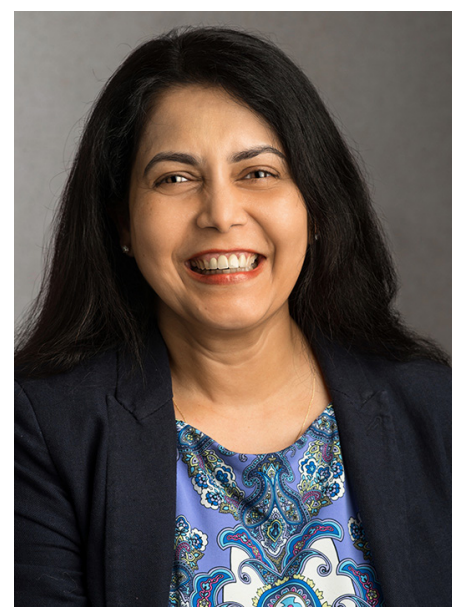

Shibani Kanungo

Shibani Kanungo

Department of Pediatric and Adolescent Medicine, Western Michigan University Homer Stryker MD School of Medicine, Kalamazoo, Michigan, USA.

(Email: Shibani.Kanungo@med.wmich.edu) doi: $10.21037 / \mathrm{atm} .2018 .12 .40$

Conflicts of Interest: The author has no conflicts of interest to declare. View this article at: http://dx.doi.org/10.21037/atm.2018.12.40

Cite this article as: Kanungo S. Inherited metabolic disorders-from genes to cell to patient to population to society and back. Ann Transl Med 2018;6(24):466. doi: 10.21037/ atm.2018.12.40 